\title{
HÁ RELAÇÃO DE HÁBITOS ORAIS DELETÉRIOS COM A TIPOLOGIA FACIAL E A OCLUSÃO DENTÁRIA?
}

\author{
Is there a relation between harmful oral habits and facial typology \\ and dental occlusion?
}

\author{
Cinthia Mara Johanns( ${ }^{(1)}$, Kelly Silvério(2), Ana Maria Furkim ${ }^{(3)}$, Irene Marchesan ${ }^{(4)}$
}

\begin{abstract}
RESUMO
Objetivo: verificar se há predominância de hábitos orais deletérios nos diferentes tipos faciais e aprofundar o estudo da relação entre os hábitos orais deletérios e a oclusão dentária. Método: foram analisados 307 prontuários de pacientes entre 2 e 59 anos de idade de ambos os sexos, encaminhados ao ambulatório do Instituto CEFAC. Foram anotados dos prontuários os hábitos orais deletérios, oclusão dentária, tipologia facial, sexo e idade. Resultados: observou-se que os hábitos com maior prevalência são: chupeta (125 indivíduos), ranger dentes (115 indivíduos) e mamadeira (102 indivíduos). Dentre as comparações estudadas, as que apresentaram diferença estatisticamente significante foram: a presença do uso da chupeta no grupo de 9 a 12 anos, quando comparado ao de 6 a 9 anos $(p=0,0269)$; presença do hábito de ranger dentes no grupo de 3 a 6 anos quando comparado ao grupo com mais de 20 anos ( $\mathrm{p}=0,0393)$; presença do hábito de ranger dentes no grupo de oclusão dentária classe I quando comparado ao classe III $(p=0,0128)$ e presença de oclusão dentária classe I no sexo feminino quando comparado ao masculino $(\mathrm{p}=0,0177)$. Conclusão: não há relação entre os hábitos orais deletérios e a tipologia facial. Entretanto, há relação entre o hábito oral deletério de ranger dentes com a oclusão dentária Classe I de Angle.
\end{abstract}

DESCRITORES: Mamadeira; Bruxismo; Oclusão Dentária; Face; Hábitos

\section{INTRODUÇÃO}

Hábito é o costume ou a prática adquirida pela repetição freqüente de um mesmo ato, que a princípio se faz de forma consciente e, posteriormente,

(1) Fonoaudióloga; Mestre em Distúrbios da Comunicação pela Universidade Tuiuti do Paraná, UTP, Curitiba, PR, Brasil.

(2) Fonoaudióloga; Docente do Departamento de Fonoaudiologia da Faculdade de Odontologia de Bauru da Universidade de São Paulo, Bauru - FOB/USP, Bauru, SP, Brasil; Doutora em Ciências pela Faculdade de Odontologia de Piracicaba da Universidade Estadual de Campinas - FOP/ UNICAMP.

(3) Fonoaudióloga; Professor Adjunto do Curso de Fonoaudiologia da Universidade Federal de Santa Catarina, Florianópolis, PR, Brasil; Doutora em Distúrbios da Comunicação Humana pela Universidade Federal de São Paulo UNIFESP.

(4) Fonoaudióloga; Diretora do CEFAC Pós-Graduação em Saúde e Educação, São Paulo, SP, Brasil; Doutora em Educação pela Universidade Estadual de Campinas Unicamp.

Conflito de interesses: inexistente de modo inconsciente. Os principais hábitos que podem suscitar deformidades na oclusão são: onicofagia, bruxismo, mordedura de objetos, mordedura de lábios, além dos mais típicos hábitos de sucção de dedo, chupeta e mamadeira ${ }^{1,2}$. Estes últimos são bastante freqüentes e tendem a perdurar, principalmente em crianças que não receberam, ou mesmo obtiveram de forma satisfatória uma amamentação natural nos seis primeiros meses de vida ${ }^{1,3,4}$.

Os hábitos orais deletérios são altamente relacionados com etiologia de más oclusões, principalmente a mordida aberta anterior ${ }^{5,6}$, posterior $^{7} \mathrm{e}$ alterações de estruturas e funções do sistema estomatognático, tais como, mastigação, deglutição, respiração e fonoarticulação8-11.

Outro fator que pode determinar má oclusão é a tipologia facial, que já é determinada, em grande parte, geneticamente, o que a torna fator importante para o diagnóstico e tratamento ortodôntico, uma vez que certos procedimentos realizados podem atenuar ou acentuar a característica facial do indivíduo 5 . 
Vários autores $6,12,13$ relataram que as alterações provocadas pelos hábitos orais deletérios irão influenciar o crescimento da face, a forma das arcadas dentárias e a produção da fala principalmente pelo padrão de posicionamento da língua. Além disso, tais alterações poderão prejudicar também as funções de mastigação, deglutição e respiração. Alertam ainda para que esses hábitos sejam considerados como risco para alterações de oclusão' ${ }^{13}$.

O conhecimento dos danos que os hábitos orais deletérios podem provocar na oclusão e no padrão facial é de suma importância para que implicações odontológicas, fonoaudiológicas e psicológicas não venham causar alterações no bom desenvolvimento da criança $a^{5,6,14,15}$.

Na prática clínica os fonoaudiólogos que atuam na área de Motricidade Orofacial questionam e cogitam a hipótese de existir uma relação entre os hábitos orais deletérios e a tipologia facial, mas não se encontra na literatura evidência científica para tais relações.

Assim, o objetivo deste estudo foi verificar se há predominância dos hábitos orais deletérios nos diferentes tipos faciais e aprofundar o estudo da relação entre os hábitos orais deletérios e a oclusão dentária.

\section{MÉTODO}

\section{Casuística}

Foram analisados 395 prontuários, destes foram excluídos 88, permanecendo no estudo 307 indivíduos entre 2 e 59 anos de ambos os sexos, encaminhados ao ambulatório de Motricidade Orofacial da clínica escola do Instituto CEFAC, por professores, dentistas, médicos, fonoaudiólogos e psicólogos. Desses 26 (8,46\%) eram braquifaciais, 110 (35,83\%) eram mesofaciais e 171 (55,71\%) dolicofaciais.

Foram excluídos da amostra, indivíduos cujos prontuários tinham dados incompletos de preenchimento, de identificação e/ou dos itens analisados na pesquisa.

\section{Procedimento}

A avaliação fonoaudiológica foi realizada por fonoaudiólogos especialistas em Motricidade Orofacial (com no mínimo 8 anos de formação) com a aplicação do protocolo MBGR ${ }^{16}$. Os indivíduos eram avaliados na primeira consulta após encaminhamento escolar, odontológico, médico, fonoaudiológico e psicológico. Os dados eram preenchidos pelo avaliador.

O protocolo de exame clínico $\mathrm{MBGR}^{16}$ compreende observação da postura corporal, análise morfológica extra e intra-oral, avaliação da mobilidade, tonicidade e sensibilidade orofacial, além das funções de respiração, mastigação, deglutição e fala.

As questões abordadas no protocolo $\mathrm{MBGR}^{16}$ são: identificação, queixas, antecedentes familiares e intercorrências; desenvolvimento e dificuldades motoras; problemas de saúde e respiratórios, sono e tratamentos realizados; aspectos ligados à alimentação desde a amamentação até a alimentação atual; bem como sobre a mastigação, deglutição, hábitos orais e também aspectos envolvendo a comunicação, fala, audição, voz e escolaridade. Abrange ainda o exame miofuncional orofacial que envolve: postura de cabeça e de ombros; medidas da face, movimento mandibular e oclusão; análise facial (avaliada com paquímetro); exame intra-oral envolvendo bochechas, língua, palato, tonsilas palatinas, dentes e oclusão; mobilidade, tonicidade e dor à palpação; além das funções de respiração, mastigação, deglutição, fala e voz.

Para a realização do presente estudo, foram selecionados dos prontuários dos indivíduos, os seguintes itens: sexo, idade, hábitos orais deletérios, oclusão e tipologia facial.

Os prontuários dos indivíduos foram divididos em grupos de faixas etárias, considerando-se o desenvolvimento da oclusão, efetuado sob o ponto de vista da observação clínica ${ }^{17}$.

Foi analisada a presença de hábitos orais deletérios de acordo com a faixa etária: 3 a 6 anos, 6 a 9 anos, 9 a 12 anos, 12 a 20 anos e acima de 20 anos.

Em seguida, foram relacionados os hábitos orais deletérios com a tipologia facial, com a oclusão dentária e com o sexo. Na seqüência foi estudada a relação entre sexo e tipologia facial, e entre sexo e oclusão dentária, segundo classificação de Angle.

Esta pesquisa foi realizada com a autorização do Comitê de Ética em Pesquisa em humanos e animais da Universidade Tuiuti do Paraná CEP-UTP, sob o protocolo de No. 000023/2009.

\section{Análise estatística}

Para análise estatística, os dados foram tabulados com os resultados das freqüências absolutas e relativas (\%) sendo então, sempre que possível utilizado o teste de diferença de proporções, adotando-se o nível de significância de 0,05 para comparação entre os grupos.

\section{RESULTADOS}

Os resultados encontram-se nas tabelas de 1 a 6 . 
Tabela 1 - Distribuição de sujeitos (em números e porcentagem) de acordo com a prevalência dos hábitos orais deletérios nos grupos de faixa etária de 3 a 6 anos, 6 a 9 anos, 9 a 12 anos, 12 a 20 anos e acima de 20 anos de idade

\begin{tabular}{|c|c|c|c|c|c|c|}
\hline $\begin{array}{l}\text { HÁBITOS ORAIS } \\
\text { DELETÉRIOS }\end{array}$ & 3 a 6 anos & 6 a 9 anos & 9 a 12 anos & 12 a 20 anos & $\begin{array}{l}\text { Acima de } \\
20 \text { anos }\end{array}$ & TOTAL \\
\hline Apertar dentes & $5(7,4 \%)$ & $20(10,0 \%)$ & $18(10,34 \%)$ & $8(9,3 \%)$ & $8(17,39 \%)$ & 59 \\
\hline $\begin{array}{l}\text { Apoiar a mão na } \\
\text { mandíbula }\end{array}$ & $1(1,4 \%)$ & $6(3,0 \%)$ & $4(2,2 \%)$ & $2(2,3 \%)$ & $1(2,1 \%)$ & 14 \\
\hline Chupeta & $12(17,91 \%)$ & $33(16,5 \%)$ & $45(25,86 \%)$ & $26(30,23 \%)$ & $9(19,56 \%)$ & 125 \\
\hline Dedo & $1(1,4 \%)$ & $10(5,0 \%)$ & $10(5,74 \%)$ & $2(2,3 \%)$ & $3(6,5 \%)$ & 26 \\
\hline Interpor lábio inferior & $3(4,4 \%)$ & $2(1,0 \%)$ & $1(0,57 \%)$ & $1(1,1 \%)$ & $0(0,0 \%)$ & 7 \\
\hline Mamadeira & $14(20,89 \%)$ & $37(18,5 \%)$ & $30(17,24 \%)$ & $17(19,76 \%)$ & $4(8,6 \%)$ & 102 \\
\hline Mascar chicletes & $0(0,0 \%)$ & $0(0,0 \%)$ & $0(0,0 \%)$ & $0(0,0 \%)$ & $0(0,0 \%)$ & 0 \\
\hline Morder a mucosa & $0(0,0 \%)$ & $5(2,5 \%)$ & $1(0,57 \%)$ & $3(3,4 \%)$ & $1(2,1 \%)$ & 10 \\
\hline Morder objetos & $1(1,4 \%)$ & $7(3,5 \%)$ & $3(1,7 \%)$ & $4(4,6 \%)$ & $1(2,1 \%)$ & 16 \\
\hline Não tem hábito & $0(0,0 \%)$ & $11(5,5 \%)$ & $6(3,4 \%)$ & $4(4,6 \%)$ & $5(10,86 \%)$ & 26 \\
\hline Ranger dentes & $20(29,85 \%)$ & $38(19,0 \%)$ & $32(18,39 \%)$ & $19(22,09 \%)$ & $6(13,04 \%)$ & 115 \\
\hline Roer unhas & $5(7,4 \%)$ & $27(13,5 \%)$ & $20(11,49 \%)$ & $5(5,8 \%)$ & $8(17,39 \%)$ & 65 \\
\hline Umidificar lábios & $5(7,4 \%)$ & $4(2,0 \%)$ & $4(2,2 \%)$ & $4(4,6 \%)$ & $0(0,0 \%)$ & 17 \\
\hline TOTAL & 67 & 200 & 174 & 86 & 46 & 573 \\
\hline
\end{tabular}

Tabela 2 - Distribuição de sujeitos (em números e porcentagem), de acordo com hábitos orais deletérios em relação à tipologia facial

\begin{tabular}{|c|c|c|c|c|c|c|}
\hline \multirow{3}{*}{$\begin{array}{l}\text { HÁBITOS ORAIS } \\
\text { DELETÉRIOS }\end{array}$} & \multirow{3}{*}{ Braquifacial } & \multirow{3}{*}{ Mesofacial } & \multirow{3}{*}{ Dolicofacial } & \multicolumn{3}{|c|}{$\begin{array}{l}\text { COMPARAÇÃO ENTRE } \\
\text { TIPOLOGIAS FACIAIS }\end{array}$} \\
\hline & & & & $\begin{array}{c}\text { Braquifacial e } \\
\text { Mesofacial } \\
\end{array}$ & $\begin{array}{c}\text { Braquifacial e } \\
\text { Dolicofacial }\end{array}$ & $\begin{array}{c}\text { Mesofacial e } \\
\text { Dolicofacial }\end{array}$ \\
\hline & & & & $\mathbf{p}$ & $\mathbf{p}$ & $\mathbf{p}$ \\
\hline Apertar dentes & $5(9,25 \%)$ & $19(10,43 \%)$ & $28(12,28 \%)$ & 0,8012 & 0,5339 & 0,5593 \\
\hline Chupeta & $12(22,22 \%)$ & $25(13,73 \%)$ & $1(0,43 \%)$ & 0,1331 & - & - \\
\hline Mamadeira & $8(14,81 \%)$ & $25(13,73 \%)$ & $30(13,15 \%)$ & 0,8409 & 0,7482 & 0,8641 \\
\hline $\begin{array}{l}\text { Apoiar a mão na } \\
\text { mandíbula }\end{array}$ & $3(5,55 \%)$ & $2(1,09 \%)$ & $7(3,07 \%)$ & - & - & - \\
\hline Morder a mucosa & $3(5,55 \%)$ & $1(0,54 \%)$ & $5(2,19 \%)$ & - & - & - \\
\hline Morder objetos & $1(1,85 \%)$ & $4(2,19 \%)$ & $8(3,50 \%)$ & - & - & - \\
\hline Ranger dentes & $12(22,22 \%)$ & $29(15,93 \%)$ & $40(17,54 \%)$ & 0,2851 & 0,4259 & 0,6653 \\
\hline Roer unhas & $5(9,25 \%)$ & $21(11,53 \%)$ & $30(13,15 \%)$ & 0,6387 & 0,4350 & 0,6216 \\
\hline Não tem hábito & $2(3,70 \%)$ & $42(23,07 \%)$ & $61(26,75 \%)$ & - & - & 0,3938 \\
\hline Umidificar lábios & $2(3,70 \%)$ & $6(3,29 \%)$ & $8(3,50 \%)$ & - & - & 0,9073 \\
\hline Dedo & $1(1,85 \%)$ & $6(3,29 \%)$ & $6(2,63 \%)$ & - & - & 0,6937 \\
\hline Interpor lábio & $0(0,00 \%)$ & $2(1,09 \%)$ & $3(1,31 \%)$ & - & - & - \\
\hline Chiclete & $0(0,00 \%)$ & $0(0,00 \%)$ & $1(0,43 \%)$ & - & - & - \\
\hline TOTAL & 54 & 182 & 228 & & & \\
\hline
\end{tabular}

Teste de diferença de proporções (nível de significância de 0,05) 
Tabela 3 - Distribuição de sujeitos (em números e porcentagem), de acordo com hábitos orais deletérios em relação à oclusão dentária

\begin{tabular}{|c|c|c|c|c|c|c|}
\hline \multirow{3}{*}{$\begin{array}{l}\text { HÁBITOS ORAIS } \\
\text { DELETÉRIOS }\end{array}$} & \multicolumn{3}{|c|}{ CLASSES } & \multicolumn{3}{|c|}{ COMPARANDO AS CLASSES } \\
\hline & \multirow{2}{*}{ I } & \multirow{2}{*}{ II } & \multirow{2}{*}{ III } & I e II & I e III & II E III \\
\hline & & & & $\mathbf{P}$ & $\mathbf{P}$ & $\mathbf{P}$ \\
\hline Apertar dentes & $24(10,61 \%)$ & $15(9,03 \%)$ & $17(11,18 \%)$ & 0,6702 & 0,8613 & 0,5347 \\
\hline Chupeta & $40(17,69 \%)$ & $35(21,08 \%)$ & $35(23,02 \%)$ & 0,3996 & 0,2034 & 0,6769 \\
\hline Mamadeira & $39(17,25 \%)$ & $33(19,87 \%)$ & $23(15,13 \%)$ & 0,5083 & 0,5855 & 0,2684 \\
\hline $\begin{array}{l}\text { Apoiar a mão na } \\
\text { mandíbula }\end{array}$ & $3(0,13 \%)$ & $4(2,40 \%)$ & $4(2,63 \%)$ & - & - & - \\
\hline Morder a mucosa & $4(0,17 \%)$ & $2(1,20 \%)$ & $3(1,97 \%)$ & - & - & - \\
\hline Morder objetos & $4(0,17 \%)$ & $6(3,61)$ & $5(3,28 \%)$ & - & - & 0,8722 \\
\hline Ranger dentes & $55(24,33 \%)$ & $29(17,46 \%)$ & $21(13,81 \%)$ & 0,1022 & $0,0128 *$ & 0,3724 \\
\hline Roer unhas & $29(12,83 \%)$ & $17(10,24 \%)$ & $21(13,81 \%)$ & 0,4316 & 0,7829 & 0,3041 \\
\hline Não tem hábito & $11(4,86 \%)$ & $8(4,81 \%)$ & $6(3,94 \%)$ & 0,9818 & 0,6722 & 0,7056 \\
\hline Umidificar lábios & $6(2,65 \%)$ & $3(1,80 \%)$ & $7(4,60 \%)$ & - & 0,3080 & - \\
\hline Dedo & $8(3,53 \%)$ & $11(6,62 \%)$ & $8(5,26 \%)$ & 0,1597 & 0,4129 & 0,6095 \\
\hline Interpor lábio & $2(, 088 \%)$ & $3(1,80 \%)$ & $2(1,31 \%)$ & - & - & - \\
\hline Chiclete & $1(0,44 \%)$ & $0(0,00 \%)$ & $0(0,00 \%)$ & - & - & - \\
\hline TOTAL & 226 & 166 & 152 & - & - & - \\
\hline
\end{tabular}

Teste de diferença de proporções (nível de significância de 0,05)

${ }^{*} p<0,05$

Tabela 4 - Distribuição de sujeitos (em números e porcentagem), de acordo com hábitos orais deletérios em relação ao sexo

\begin{tabular}{lccc}
\hline HÁBITOS ORAIS DELETÉRIOS & FEMININO & MASCULINO & Valor de $\mathbf{p}$ \\
\hline Apertar dentes & $27(11,63 \%)$ & $32(8,9 \%)$ & 0,2802 \\
Chupeta & $50(21,55 \%)$ & $74(20,67 \%)$ & 0,7978 \\
Mamadeira & $35(15,08 \%)$ & $68(18,99 \%)$ & 0,2221 \\
Apoiar a mão na mandíbula & $7(3,01 \%)$ & $8(2,2 \%)$ & 0,5399 \\
Morder a mucosa & $5(2,15 \%)$ & $7(1,9 \%)$ & 0,8322 \\
Morder objetos & $8(3,44 \%)$ & $6(1,6 \%)$ & 0,1478 \\
Ranger dentes & $42(18,10 \%)$ & $72(20,11 \%)$ & 0,5460 \\
Roer unhas & $29(12,5 \%)$ & $47(13,12 \%)$ & 0,8262 \\
Não & $10(4,31 \%)$ & $14(3,9 \%)$ & 0,8054 \\
umidificar lábios & $6(2,5 \%)$ & $10(2,7 \%)$ & 0,8820 \\
Dedo & $11(4,7 \%)$ & $14(3,9 \%)$ & 0,6368 \\
Interpor lábio inferior & $2(8,86 \%)$ & $5(1,3 \%)$ & - \\
Mascar chiclete & $0(0,00 \%)$ & $1(0,2 \%)$ & - \\
\hline TOTAL & 232 & 358 & \\
\hline
\end{tabular}

Teste de diferença de proporções (nível de significância de 0,05) 
Tabela 5 - Prevalência do sexo masculino e feminino em relação à tipologia facial

\begin{tabular}{lcccc}
\hline SEXO & BRAQUIFACIAL & DOLICOFACIAL & MESOFACIAL & TOTAL \\
\hline FEMININO & $12(10,26 \%)$ & $70(59,83 \%)$ & $35(29,91 \%)$ & $117(100 \%)$ \\
MASCULINO & $14(7,37 \%)$ & $101(53,16 \%)$ & $75(39,47 \%)$ & $190(100 \%)$ \\
\hline Valor de p & 0,3778 & 0,2541 & 0,0908 & \\
\hline
\end{tabular}

Teste de diferença de proporções (nível de significância de 0,05)

Tabela 6 - Prevalência do sexo masculino e feminino em relação à oclusão dentária

\begin{tabular}{|c|c|c|c|}
\hline OCLUSÃO DENTÁRIA & MASCULINO & FEMININO & Valor de $p$ \\
\hline Classe I & $68(35,78 \%)$ & $58(49,57 \%)$ & $0,0177^{*}$ \\
\hline Classe II & $58(30,52 \%)$ & $24(20,51 \%)$ & 0,0551 \\
\hline Classe II 1a & $0(0,00 \%)$ & $1(0,85 \%)$ & - \\
\hline Classe II $2^{\underline{a}}$ & $2(1,05 \%)$ & $0(0,00 \%)$ & - \\
\hline Classe III & $43(22,63 \%)$ & $31(26,49 \%)$ & 0,4431 \\
\hline Classe IE IID & $6(3,15 \%)$ & $0(0,00 \%)$ & - \\
\hline Classe ID IIE & $1(0,50 \%)$ & $1(0,85 \%)$ & - \\
\hline Classe ID IIIE & $2(1,05 \%)$ & $0(0,00 \%)$ & - \\
\hline Classe IE IIID & $3(1.57 \%)$ & $0(0,00 \%)$ & - \\
\hline Molar não erupcionado & $7(3,68 \%)$ & $2(1,70 \%)$ & - \\
\hline TOTAL & 190 & 117 & \\
\hline
\end{tabular}

Teste de diferença de proporções (nível de significância de 0,05)

${ }^{*} p<0,05$

\section{DISCUSSÃO}

De acordo com os resultados deste trabalho, os hábitos orais deletérios mais prevalentes foram, uso de chupeta no grupo de 12 a 20 anos (30,23\%), ranger dentes no grupo de 3 a 6 anos $(29,85 \%)$, ressaltando que os indivíduos relataram apresentar mais de um hábito oral deletério (Tabela 1).

No presente estudo, o hábito de ranger dentes mais encontrado na população com idade entre 3 e 6 anos pode estar relacionado ao alto nível de stress encontrado em crianças. Alguns autores ${ }^{2}$ não encontraram relação entre bruxismo e fatores oclusais ou entre bruxismo e hábitos orais como onicofagia, embora tenham encontrado relação de bruxismo com uso de chupeta.

Por outro lado, indivíduos com idade entre 12 e 20 anos relataram ter usado chupeta durante muitos anos. Apesar das razões para o uso prolongado da chupeta não terem sido pesquisadas, uma das hipóteses poderia ser a falta de conhecimento dos possíveis malefícios que a mesma pode causar.

Ao analisar a relação entre a duração do aleitamento materno, alimentação artificial e hábitos de sucção foi observado que a duração do aleitamento artificial foi positivamente correlacionada com a duração de hábitos de sucção, portanto, quanto mais tempo a criança é amamentada, menor a chance de apresentar hábitos orais deletérios de sucção ${ }^{18}$.

Os hábitos orais deletérios não só prejudicam a oclusão, como também podem prejudicar a fala. Essa foi a conclusão de um estudo que investigou a associação do uso da mamadeira, chupeta e outros hábitos de sucção com distúrbios da fala, onde se concluiu que o habito de sucção prolongado pode ter efeitos prejudiciais sobre o desenvolvimento da fala ${ }^{19}$.

Outro estudo concluiu que a maioria das crianças apresentava o hábito de sucção não nutritiva até os 24 meses de vida, o qual foi associado com a má oclusão ${ }^{20}$.

No presente estudo, quando comparados os hábitos orais deletérios em relação à tipologia facial, foi possível observar que não houve diferença significante quando comparados os tipos faciais em relação aos hábitos orais deletérios. No entanto, os braquifaciais foram os indivíduos que tiveram o menor número de hábitos 14,5\% (52) e os dolicofaciais o maior $46,5 \%$ (167) seguido dos mesofaciais 39\% (140). 
Dentre aqueles hábitos que os fonoaudiólogos clínicos mais têm preocupação, observou-se que o hábito oral deletério de chupeta $(22,22 \%)$, e ranger dentes $(22,22 \%)$ foi o mais freqüente no tipo braquifacial assim como o hábito de roer unhas foi encontrado mais nos dolicofaciais $(13,15 \%)$. A sucção de dedo foi pouco encontrada, mas esteve mais presente nos mesofaciais $(3,29 \%)$. O hábito de uso da mamadeira, embora mais presente nos braquifaciais $(14,81 \%)$, esteve muito próximo dos valores encontrados para os mesofaciais $(13,73 \%)$ e para os dolicofaciais (13,15\%). A ausência total de hábitos foi relatada em $26,75 \%$ para dolicofaciais, $23,07 \%$ para mesofaciais e $3,70 \%$ para braquifaciais (Tabela 2).

Os achados deste estudo demonstram que não houve relação entre hábitos orais deletérios e tipologia facial. Os fonoaudiólogos clínicos de uma maneira em geral acreditam que os hábitos deletérios interferem fortemente no crescimento craniofacial.

Autores de um estudo epidemiológico sobre padrão facial na dentadura decídua ${ }^{21}$ encontraram predomínio de crianças mesofaciais $(64,56 \%)$ em relação às dolicofaciais $(21,90 \%)$ e braquifaciais $(13,54 \%)$. Nesse estudo, por ter sido realizado a partir de um banco de dados de uma clínica, o tipo dolicofacial foi o mais encontrado $55,71 \%$ (171) seguido dos mesofaciais $35,83 \%$ (110) e pelos braquifaciais $8,46 \%$ (26).

Observa-se na Tabela 3, o estudo da relação entre os hábitos orais deletérios com a oclusão dentária. Verificou-se que ocorreu diferença significante no hábito oral deletério de ranger dentes entre as classes I e III ( $p=0,0128)$, mostrando que a proporção deste hábito oral deletério entre os indivíduos com classe I $(24,33 \%)$ é significantemente superior entre indivíduos com classe III (13,81\%).

Alguns estudos exploraram a relação entre hábitos orais deletérios e a oclusão ${ }^{13,22-24}$ e constataram a existência de associação entre esses fatores. Alguns observaram relação direta sem, no entanto, especificar quais ${ }^{13,23,24}$, outro observou predomínio do uso da mamadeira em crianças classe I ${ }^{22}$; e também foi observada relação significante entre hábitos de sucção e protrusão dos incisivos superiores e inferiores ${ }^{25}$. Esses achados, concordam com os estudos ${ }^{13,24}$ que verificaram que das crianças portadoras de hábitos orais deletérios, 87\% apresentavam má oclusão.

Cabe ressaltar, que intervenções preventivas neste caso, seriam de grande valia. O conhecimento da etiologia da má oclusão é essencial, porque a sua correção requer a eliminação das causas. Em todas as classificações sobre a etiologia das más oclusões, os hábitos orais deletérios foram mencionados como um agente em potencial para o seu desenvolvimento $3,9,20,24,25-28$. Neste sentido, pode-se assumir que uma das causas dos problemas oclusais é a persistência prolongada dos hábitos em fases da infância posteriores ao quarto ano de vida.

A Tabela 4 revela o estudo da relação entre a prevalência dos hábitos orais deletérios e o sexo, evidenciando que não houve diferença significante entre os mesmos. Porém, $21,55 \%$ dos indivíduos do sexo feminino e $20,67 \%$ do masculino, apresentavam o hábito oral deletério de chupar chupeta, sendo este, o mais apresentado na amostra.

$\mathrm{Na}$ Tabela 5 encontra-se o estudo da relação entre a prevalência do sexo masculino e feminino e a tipologia facial. Verificou-se que não ocorreu diferença significante. Entretanto, o tipo facial mais encontrado nesta amostra é o tipo dolicofacial ( $59,83 \%$ do sexo feminino e $53,16 \%$ do masculino). Em uma pesquisa realizada com 2.009 crianças $^{21}$ onde as mesmas foram classificadas de acordo com o padrão facial, encontrou-se que 1.270 $(63,22 \%)$ foram consideradas padrão facial equilibrado (mesofacial), $665(33,10 \%)$ padrão facial longo (dolicofacial) e 74 (3,68\%) padrão facial curto (braquifacial). Em relação ao gênero, 1.027 $(51,11 \%)$ crianças eram do sexo masculino e 982 $(48,88 \%)$ do sexo feminino, não ocorrendo diferença estatisticamente significante. Dessa forma, os dados do presente estudo, concordam com estudos que também não observaram diferenças estatísticas entre os gêneros ${ }^{21,24}$. É importante ressaltar que pode haver diferença nos dados quando consideradas outras regiões ou países.

A Tabela 6 mostra o estudo da relação entre a prevalência do sexo masculino e feminino com a oclusão dentária. Verificou-se que ocorreu diferença significante entre as proporções de indivíduos do sexo masculino e feminino, na classe I, ou seja, a proporção de indivíduos do sexo feminino com classe I é significantemente superior aos do sexo masculino $(p=0,0177)$. É importante salientar que houve uma tendência dos indivíduos do sexo masculino a apresentar mais oclusão classe II do que os indivíduos do sexo feminino $(\mathrm{p}=0,0551)$. Para alguns autores ${ }^{29}$, a maioria dos indivíduos estudados apresentou Classe II, seguido de Classe I e, por fim, Classe III, independentemente do gênero.

A adoção de procedimentos de orientação tem se mostrado benéfica para promover a interrupção dos hábitos orais deletérios ${ }^{30}$.

Para melhor solução dos problemas dos hábitos orais deletérios, devem-se criar políticas que envolvam o esclarecimento da família do paciente, já que a sua erradicação precoce depende da 
cumplicidade familiar, o que pode ser alcançado por meio de campanhas educativas que promovam a saúde integral do indivíduo.

Finalizando, há necessidade de mais estudos sobre os hábitos orais deletérios e sua relação com a tipologia facial, abordando diferentes hábitos e sua relação com a oclusão dentária, nas diferentes faixas etárias.

Convém sugerir que pesquisas na área fonoaudiológica direcionadas à motricidade orofacial, continuem sendo realizadas, para que sejam desenvolvidas técnicas mais específicas e meios de tratamento mais eficazes, buscando uma padronização dos resultados.
Por fim, devido à evidência de hábitos que a literatura classifica como reflexos do mundo moderno na vida psicológica das crianças sugerese, também, a realização de pesquisas com populações homogêneas a fim de se verificar as relações existentes entre o estresse infantil e hábitos orais deletérios.

\section{CONCLUSÃO}

Conclui-se que não há predominância dos hábitos orais deletérios nos diferentes tipos faciais. No entanto, este estudo encontrou relação significante entre o hábito oral deletério de ranger dentes com a oclusão dentária classe I de Angle.

\begin{abstract}
Purpose: to check whether there is a predominance of deleterious oral habits in different facial types and further study of the relationship between harmful oral habits and dental occlusion. Method: we analyzed 307 cases of patients between 2 and 59-year old of both genders, referred to the outpatient clinic of CEFAC Institute. Were we registered medical records of deleterious oral habits, dental occlusion, facial type, gender and age. Results: we observed that the following habits are most prevalent: pacifier (125 subjects), gnashing teeth (115 subjects) and bottle (102 individuals). Among the comparisons, the ones who showed statistical significance was the presence of pacifier use in the group from 9 to 12 years, compared to 6 to 9 years $(p=0.0269)$, presence of habit of gnashing teeth in the group from 3 to 6 years when compared to more than 20 years $(p=0.0393)$, presence of the habit of gnashing teeth in the group of dental occlusion class I compared to class III $(p=0.0128)$ and presence dental occlusion class I in females compared to males $(p=0.0177)$. Conclusion: there is no relationship between harmful oral habits and facial typology. However, there is a relationship between the harmful oral habit of grinding the teeth with Angle class I dental occlusion.
\end{abstract}

KEYWORDS: Nursing Bottle; Bruxism; Dental Occlusion; Face; Habits

\section{REFERÊNCIAS}

1. Valença AMG, Vasconcelos FGG, Cavalcanti AL, Duarte RC. Prevalência e características de hábitos orais em crianças. Pesqui Bras Odontoped Clin Integr. 2001; 1(1):17-24.

2. Gonçalves LPV,Toledo OA, Otero SAM. Relação entre bruxismo, fatores oclusais e hábitos bucais. Dental Press J. Orthod. 2010; 15(2) 97-104.

3. Caglar E, Larsson E, Andersson EM, Hauge MS, Ogaard B, Bishara S, Warren J, Noda T, Dolci GS. Feeding, artificial sucking habits, and malocclusions in 3-year-old girls in different regions of the world. $J$ Dent Child. 2005; 72(1): 25-30.

4. Gimenez CMM, Moraes ABA, Bertoz AP, Bertoz FA, Ambrosano GB. Prevalência de más oclusões na primeira infância e sua relação com as formas de aleitamento e hábitos infantis. Rev. Dent. Press Ortodon. Ortopedi. Facial. 2008; 13(2):70-83.

5. Heimer MV, Tornisiello Katz CR, Rosenblatt A. Non-nutritive sucking habits, dental malocclusions, and facial morphology in Brazilian children: a longitudinal study. 2008; Eur J Orthod. 30(6):580-5. 6. Ovsenik M. Incorrect orofacial functions until 5 years of age and their association with posterior crossbite. Am J Orthod Dentofacial Orthop. 2009; 136(3):375-81.

7. Melink S, Vagner MV, Hocevar-Boltezar I, Ovsenik M. Posterior crossbite in the deciduous dentition period, its relation with sucking habits, irregular orofacial functions, and otolaryngological findings. Am J Orthod Dentofacial Orthop. 2010; 138(1):32-40.

8. Warren JJ, Bishara ES, Kari LS et al. Effects of oral habits duration on dental characteristics in 
the primary dentition. J Amer dent Assoc. 2001; 13(12):1685-93.

9. Chevitarese AB, Della Valle D, Moreira TC. Prevalence of malocclusion in 4-6 year old Brazilian children. J Clin Pediatr Dent. 2002; 27(1): 81-6.

10. Bertoldi P, Felício M, Matsumoto MA. Effect of the early intervention of oral habits on the development of dental occlusion. Pró-fono Rev Atualização Científica. 2005; 17(1): 37-44.

11. Sahad MG, Nahás ACR, Scavone-Junior $H$, Jabur LB, Guedes-Pinto E. Vertical interincisal trespass assessment in children with speech disorders. Braz Oral Res. 2008; 22(3):247-51.

12. Peres KG, Barros AJD, Peres MA, Cictora CG. Effects of breastfeeding and sucking habits on malocclusion in a birth cohort study. Revista de saude Publica. 2007; 41(3): 343-50.

13. Leite-Cavalcanti A, Bezerra PKM, Moura C. Aleitamento natural, aleitamento artificial, hábitos de sucção e maloclusões em pré-escolares brasileiros. Rev Salud Publica. 2007; 9(2):194-204.

14. Kulkarni GV, Lau D. A single appliance for the correction of digit-sucking, tongue-thrust, and posterior cross bite. Pediatr Dent. 2010; 32(1):61-3. 15. Pereira ERBN, Trezza EMC. Identificação das atitudes dos pais e familiares frente ao uso da chupeta. J Bras Fonoaudiol. 2005; 5(23):381-6.

16. Genaro KF, Berretin-Felix G, Rehder MIBC, Marchesan IQ. Avaliação Miofuncional Orofacial Protocolo MBGR. Rev. CEFAC. 2009; 11(2):237-55. 17. Petrelli E. Ortodontia para fonoaudiologia. Editora Lovise: Curitiba; 1994.

18. Medeiros AP, Ferreira JT, Felicio CM. Correlation between feeding methods, non-nutritive sucking and orofacial behavior. Pro Fono. 2009; 21(4): 315-9.

19. Barbosa C, Vasquez S, Parada MA, Gonzalez JC, Jackson C, Yanez ND, Gelaye B, Fitzpatrick AL. The relationship of bottle feeding and other sucking behavior with speech disorder in Patagonian preschoolers. BMC Pediatr. 2009; 9:66.

20. Duncan K, McNamara C, Ireland AJ, Sandy JR. Sucking habits in childhood and the effects on the primary dentition: findings of the Avon longitudinal study of pregnancy and childhood. Int J Pedit Dent. 2008; 18:178-88.

21. Silva Filho OG, Herkrath FJ, Queiroz APC, Aiello CA. Padrão facial na dentadura decídua: estudo epidemiológico. R Dental Press Ortodon Ortop Facial. 2008; 13(4):45-59.

22. Almeida FL, Silva AMT, Serpa EO. Relação entre má oclusão e hábitos em respiradores orais. Rev CEFAC. 2009; 11(1):86-93.

23. Onyeaso CO, Isiekwe MC. Oral habits in the primary and mixed dentitions of some Nigerian children: a longitudinal study. Oral Health Prev Dent. 2008; 6(3):185-90.

24. Katz CR, Rosenblatt A, Gondim PP. Nonnutritive sucking habits in brazilian children: effects on deciduous dentition and relationship with facial morphology. Am J Orthod Dentofacial Orthop. 2004; 126(1): 53-7.

25. Warren JJ, Bishara SE. Duration of nutritive and nonnutritive sucking behaviors and their effects on the dental arches in the primary dentition. Am J Orthod Dentofacial Orthop. 2002; 121(4): 347-56. 26. Aznar T, Galán AF, Marin I, Domínguez A. Dental arch diameters and relationships to oral habits. Angle Orthodontist. 2005; 76(3): 441-5.

27. Bishara SE, Warren JJ, Levyd SM. Changes in the prevalence of nonnutritive sucking in the first 8 years of life. Am J Orthod Dentofac. 2006; 130(1): 31-5.

28. Katz CRT, Rosenblat A. Nonnutritive sucking habits and anterior open bite in Brazilian children. A longitudinal study. Pediatr dent. 2005; 27(5): 369-73.

29. Oliveira CF, Busanello AR, Silva AMT. Ocorrência de má oclusão e distúrbio articulatório em crianças respiradoras orais de escola pública de Santa Maria, RG. RGO. 2008; 56(2):169-74.

30. Degan VV, Puppin-Rontani RM. Removal of sucking habits and myofunctional therapy: establishing swallowing and tongue rest position. Pró-Fono. 2005; 17(3): 375-83.

ACEITO EM: 21/01/2011

Endereço para correspondência:

Kelly C. A. Silverio

Al. Dr. Octávio Pinheiro Brisolla, 9-75

Bauru - SP

CEP:17012-101

E-mail: kcsilver@terra.com.br

kellysilverio@usp.br 